\title{
Distribution of clock correction and ephemeris parameters in broadcast navigation messages
}

\author{
Ivan Lapin ${ }^{1}\left(\mathbb{D} \cdot\right.$ Jaron Samson ${ }^{2} \cdot$ Stefan Wallner ${ }^{3} \cdot$ Carlos Lopez $^{2} \cdot$ Mikael Mabilleau $^{4}$
}

Received: 19 December 2020 / Accepted: 3 June 2021 / Published online: 11 June 2021

(c) The Author(s) 2021

\begin{abstract}
The distribution of clock correction and ephemeris (CCE) parameters in navigation messages broadcast by operational satellite vehicles (SVs) of the Global Navigation Satellite System (GNSS) is studied. For this purpose, two evaluation metrics are proposed, namely atypical navigation message structures and time to complete CCE sets (TTCS). The evaluation metrics are used to analyze three and a half years of operational Galileo F/NAV and GPS LNAV messages. The study considers arbitrary and healthy-only signal-in-space (SIS) health statuses. The frequently used receiver independent exchange format (RINEX) navigation files are shown to not provide sufficient information to determine the CCE parameters transmitted at every time instance. Due to the identified limitations, binary navigation data collected by a worldwide network of monitoring stations are used for the analysis. During operations, the maximum of the TTCS metric is shown to be impacted by the issue of data (IOD) update periods and structures with mismatched IOD parameters, potentially introducing an additional delay to the time to first fix (TTFF). The message timing requirements of dual-frequency multi-constellation (DFMC) satellite-based augmentation system (SBAS) are shown to be in line with the minimum times needed to obtain multiple complete Galileo F/NAV and GPS LNAV CCE sets.
\end{abstract}

Keywords Binary navigation data $\cdot$ Clock correction and ephemeris $\cdot$ DFMC $\cdot$ GNSS navigation message $\cdot$ SBAS

\section{Introduction}

Satellite vehicles (SVs) of the Global Navigation Satellite System (GNSS) continuously broadcast navigation messages on the signal-in-space (SIS). Navigation messages contain regularly updated sets of clock correction and ephemeris (CCE) parameters allowing the users to compute their navigation solution. The real-time positioning users that depend on the broadcast CCE sets are single and dual-frequency standard positioning users or users of the safety-of-life (SoL) applications like satellite-based augmentation system

Ivan Lapin

ivan.lapin@esa.int

1 Radio Frequency Systems Division, European Space Agency, Keplerlaan 1, 2201 AZ Noordwijk, The Netherlands

2 EGNOS Project Office, European Space Agency, 18 Avenue Edouard Belin, 31401 Toulouse, France

3 Galileo Project Office, European Space Agency, Keplerlaan 1, 2201 AZ Noordwijk, The Netherlands

4 European GNSS Agency, Janovského 438/2, 17000 Prague, Czech Republic
(SBAS), ground-based augmentation system (GBAS), or receiver autonomous integrity monitoring (RAIM) (Kaplan and Hegarty 2017).

The accuracy of the disseminated CCE parameters has been already widely studied as an important performance aspect of the GPS LNAV message (Warren and Raquet 2003; Cohenour and Graas 2011; Heng et al. 2012a), GPS CNAV message (Steigenberger et al. 2015; Wang et al. 2019), GLONASS constellation (Heng et al. 2012b; Gunning et al. 2015), Galileo constellation (Perea et al. 2017; Wu et al. 2020) and Beidou constellation (Wu et al. 2017; Lv et al. 2019). A multi-GNSS framework presented by Montenbruck et al. (2018) has been used to show the SIS range error performance of all four major GNSS constellations. However, the distribution of the CCE parameters in the operational navigation messages remains insufficiently explored. The distribution of the CCE parameters impacts the time needed to receive a complete CCE set, contributing to the time to first fix (TTFF) in cold and warm receiver starts (Paonni et al. 2010). The TTFF is an important performance metric of GNSS receivers as it describes the time elapsed from the moment the receiver is started up until 
the moment when the navigation processor obtains the first position fix. So far, it has been assumed that only the repetition interval of the CCE sets and the length of the decoding words drive the time needed to obtain a complete set (Paonni et al. 2010; Delépaut et al. 2020). Additional delays caused by the operational distribution of the CCE parameters have not been considered.

Every set of the CCE parameters on a given SIS is uniquely identified by the value of the issue of data (IOD) parameter. To provide their services, some GNSS applications, such as SBAS, broadcast additional clock and ephemeris corrections to augment the CCE sets and use the value of the IOD parameter to link the corrections unambiguously. The future dual-frequency multi-constellation (DFMC) SBAS will enable dual-frequency positioning and allow system designers and service providers to include augmentation of Galileo, GPS, BeiDou, and GLONASS constellations. The future European DFMC SBAS, named European Geostationary Navigation Overlay Service (EGNOS) V3, considers augmentation of the Galileo and GPS constellations for the avionics users. As a consequence, EGNOS V3 will augment the Galileo F/NAV and GPS LNAV messages. In this context, the potential impact of the statistical distribution of the operational values of the Galileo F/NAV IOD ${ }_{\text {nav }}$ parameter on DFMC SBAS was analyzed (Lapin et al. 2018). A detailed analysis of the times needed to obtain complete CCE sets based on binary data has not yet been performed.

The distribution of the CCE parameters in broadcast navigation messages is studied and discussed. A generic model of a navigation message is formulated, and evaluation metrics are proposed. The frequently used receiver independent exchange format (RINEX) (RTCM-SC104 2018) is assessed from the perspective of determining the CCE parameters and SIS health status at every time instance. Three and a half years of operational Galileo F/NAV and GPS LNAV messages are analyzed using the proposed evaluation metrics.

\section{Broadcast navigation message methodology}

Broadcast navigation messages contain important information such as the CCE parameters needed to compute the navigation solution or the SIS health status that constrains the usage of the SV. The structure and content of broadcast navigation messages transmitted on the SIS depend on the constellation and signal component. Galileo F/NAV and GPS LNAV messages are further considered due to their relevance to DFMC SBAS.

\section{Galileo F/NAV message}

Galileo SVs broadcast F/NAV message data as a continuous stream of $600 \mathrm{~s}$ long F/NAV frames (European Union
(2020)). The F/NAV frame is composed of twelve F/NAV subframes, each taking $50 \mathrm{~s}$ to transmit. The F/NAV subframe is further composed of five $10 \mathrm{~s}$ long F/NAV pages. The structure of the F/NAV page is uniquely identified by its page type. The complete set of CCE parameters is contained in page types $1,2,3$, and 4 . The CCE set is identified by a parameter called $\mathrm{IOD}_{\text {nav }}$. Although the higher hierarchical structure of the Galileo F/NAV page is the F/NAV subframe, there are no management data transmitted within the F/NAV message to indicate subframe and frame structures (European Union (2020)). As further clarified in the interface specification, the higher-level structures should be considered as the typical flow of pages reflecting the current Galileo navigation message design, which may evolve together with the future evolutions of the system. This implies that the F/NAV pages can be transmitted in an arbitrary order and without any guaranteed repetition interval. The user is thus requested to always decode the page type identifier. The minimum achievable duration needed to obtain a complete CCE set in the F/NAV subframe structure is $40 \mathrm{~s}$. The structure of a typical F/NAV subframe containing a complete CCE set is shown in Fig. 1. The SIS health status is determined through three SIS status flags that are encoded in the F/NAV page type 1 (European Union 2019): signal health status (SHS), data validity status (DVS), and signal-in-space accuracy (SISA).

\section{GPS LNAV message}

GPS SVs broadcast LNAV message data as a continuous stream of $30 \mathrm{~s}$ long LNAV frames (U.S. Department of Defense 2013). The LNAV frame is composed of five LNAV subframes, each taking $6 \mathrm{~s}$ to transmit, and the subframe is composed of ten LNAV words. The subframe ID number uniquely identifies the structure of the LNAV subframe. The complete set of CCE parameters is contained in three LNAV subframes with IDs numbered 1,2, and 3. The CCE set is identified by two parameters: (i) issue of data, clock (IODC), which indicates the issue number of the data set, and (ii) issue of data, ephemeris (IODE), which indicates any change

\section{Complete CCE set (40s)}

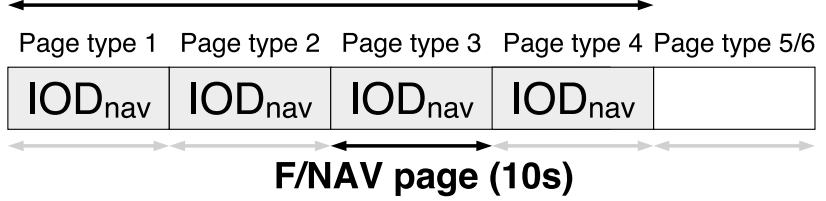

Fig. 1 Structure of a typical Galileo F/NAV subframe containing a complete $\mathrm{CCE}$ set identified by the $\mathrm{IOD}_{\text {nav }}$ parameter in page types $1,2,3$, and 4. Depending on whether the subframe is even or odd, page type 4 is typically followed by either page type 5 or 6 , which is indicated as $5 / 6$ 
in the ephemeris representation parameters. IODEs are provided in LNAV subframes 2 and 3 to compare them against the 8 least significant bits of IODC provided in LNAV subframe 1 . Whenever these three terms do not match, a data set cutover has occurred, and the user must collect new data. U.S. Department of Defense (2020) refers to the rigid higher-level structure of the LNAV subframe as the fixed frame structure, where every LNAV subframe is broadcast by every SV at the same time each week. The minimum achievable duration needed to obtain a complete CCE set is $18 \mathrm{~s}$. The fixed frame structure of the LNAV frame is shown in Fig. 2. The SIS health status is determined by considering the C/A-code signal alarms, user range accuracy (URA) alert flag, health status field, and URA parameter (U.S. Department of Defense 2020).

\section{Generic model of navigation messages}

Although different navigation messages define custom nomenclatures and structures, common logical principles can be observed and extracted to formulate a generic model. The basic recognizable data structure of every navigation message is referred to as an element. The elements of the Galileo F/NAV and GPS LNAV messages are the F/NAV pages and LNAV subframes, respectively. The specific binary structure of an element is identified by a field referred to as the element number. This number is called page type in the F/NAV message and subframe ID in the LNAV message. The CCE parameters of a complete CCE set might be transmitted in several elements with specific element numbers. In the case of the F/NAV message, the user must collect element numbers 1-4 to obtain a complete CCE set. In the case of the LNAV message, a complete set requires element numbers 1,2 , and 3 . To allow the user to match different elements into a common CCE set, an additional parameter, referred to as the IOD parameter, is present. This parameter is called $\mathrm{IOD}_{\text {nav }}$ in the F/NAV message and IODE in the LNAV message. Each element logically belongs to a higher message structure. The higher message structure of the F/ NAV page and F/NAV subframe is the F/NAV subframe and LNAV frame, respectively. The description of both messages using the proposed generic model is summarized in

\section{Complete CCE set (18s)}

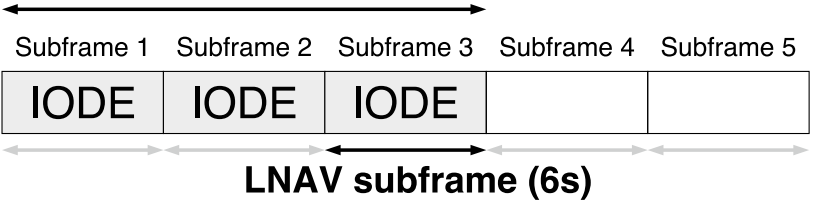

Fig. 2 Fixed frame structure of GPS LNAV frame containing a complete CCE set identified by the IODE parameter in subframes 1, 2, and 3
Table 1 . The model can be applied to other GNSS navigation messages.

\section{Evaluation metrics}

A suboptimal distribution of the CCE parameters in the SIS navigation data stream may lead to the unavailability of the CCE sets at the receiver. Therefore, evaluation metrics that allow studying this distribution are proposed.

\section{Atypical navigation message structures}

To define the atypical navigation message structures, an example of a typical F/NAV subframe is first shown in Fig. 3 (top left). The subframe provides a complete CCE set as it contains all element numbers in the correct order $(1-5$ or $1,2,3,4,6)$ with matching IOD parameters. An equivalent typical structure of the LNAV message may be imagined. Three atypical navigation message structures are of concern: the structure with missing element numbers, the structure with wrongly ordered elements, and the structure with mismatched IOD parameters.

The structure with missing element numbers does not contain all elements needed to obtain a complete CCE set. An example of the F/NAV subframe missing page type 4 is shown in Fig. 3 (top right), where page type 1 is transmitted instead. In the case of the structure with wrongly ordered elements, its elements are not ordered correctly, even if a complete CCE set can be obtained. An example of this is shown in Fig. 3 (bottom left), where the F/NAV pages do not follow the typical flow. The structure with mismatched IOD parameters contains elements whose CCE parameters do not belong to the same set. An example of a subframe with mismatched $\mathrm{IOD}_{\text {nav }}$ parameters is shown in Fig. 3 (bottom right), where the first two page types contain $\mathrm{IOD}_{\text {nav }} 1$ and the latter two page types contain mismatched $\mathrm{IOD}_{\text {nav }} 2$.

Table 1 Description of Galileo F/NAV and GPS LNAV messages using the proposed generic model

\begin{tabular}{lll}
\hline Constellation & Galileo & GPS \\
\hline Navigation message & F/NAV & LNAV \\
Signal component & E5a-I & L1C/A \\
IOD parameter & IOD $_{\text {nav }}$ & IODE \\
Element & F/NAV page & LNAV subframe \\
Element duration $[\mathrm{s}]$ & 10 & 6 \\
Element number & Page type & Subframe ID \\
Element numbers of a CCE set & $1,2,3,4$ & $1,2,3$ \\
Higher structure & F/NAV subframe & LNAV frame \\
Min. CCE duration $[\mathrm{s}]$ & 40 & 18 \\
\hline
\end{tabular}




\begin{tabular}{|l|l|l|l|l|}
\hline IOD $_{\text {nav } 1}$ & IOD $_{\text {nav } 1}$ & IOD $_{\text {nav } 1}$ & IOD $_{\text {nav } 1}$ & \\
\hline Page type 1 & Page type 2 & Page type 3 & Page type 4 & Page t. 5/6 \\
\hline
\end{tabular}

IOD nav 1 IOD nav 1 IOD nav 1 IOD nav 1

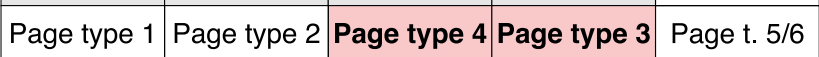

\begin{tabular}{|l|l|l|l|l|}
\hline $\operatorname{IOD}_{\text {nav }} 1$ & $\operatorname{IOD}_{\text {nav }} 1$ & $\operatorname{IOD}_{\text {nav }} 1$ & $\operatorname{IOD}_{\text {nav }} 1$ & \\
\hline Page type 1 & Page type 2 & Page type 3 & Page type 1 & Page t. 5/6 \\
\hline
\end{tabular}

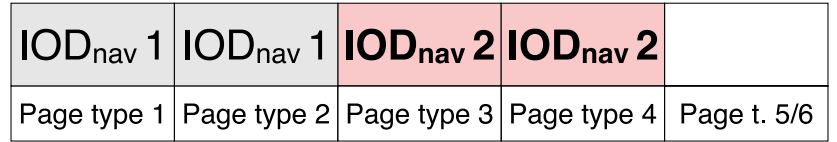

Fig. 3 Typical Galileo F/NAV subframe (top left), hypothetical subframe with missing element numbers (top right), hypothetical subframe with wrongly ordered elements (bottom left), and hypothetical subframe with mismatched IOD $_{\text {nav }}$ parameters (bottom right)

\section{Time to complete clock correction and ephemeris sets}

The time to $n$ complete CCE sets $\left(\mathrm{TTCS}_{n}\right.$ ) is defined as the time elapsed from the start of the reception until the moment a given number of unique and complete sets are first obtained by the receiver. Unique sets imply that their IOD parameters differ. No impact of the local user environment is assumed. An example of TTCS $n$ for $n$ equal to 1 and 2 in the Galileo F/NAV navigation data stream under a typical start of the reception of page type 1 with $\mathrm{IOD}_{\text {nav }} 1$ is shown in Fig. 4. Typically, the user would not start receiving the navigation data at the exact moment of the start of the first bit of an element. The typical start is considered to result in the element not being obtained and delays the completion of the CCE set until the element is received in full. Since the reception begins $7.5 \mathrm{~s}$ after the start of page type 1 in the example shown in Fig. 4, this page is not obtained, and the reception is delayed until page type 1 is transmitted again. Collecting all necessary page types $(1,2,3$, and 4$)$ of the set with $\operatorname{IOD}_{\text {nav }} 1$ thus takes TTCS $_{1}=52.5 \mathrm{~s}$. The next unique CCE set in the given example stream is identified by $\operatorname{IOD}_{\text {nav }} 2$. The time needed to complete it for the first time from the assumed start of the reception takes TTCS $_{2}=182.5 \mathrm{~s}$.

\section{Minimum time to multiple complete clock correction and ephemeris sets}

For some applications, understanding the minimum periods during which a given number of unique and complete CCE sets may be received is important. For example, SBAS users and systems need to consider the message timing requirements that are defined in the applicable standardization documents. DFMC SBAS minimum operational performance standards (MOPS) (Eurocae 2021) specifies the minimum number of sets that the SBAS user receiver is required to store in its memory per each SV of a given GNSS constellation. DFMC Standards and Recommended Practices (SARPs) (ICAO 2018) define how long the SBAS receiver should wait before augmenting a newly issued CCE set by the SV. The proper synergy between these two requirements and the operational GNSS constellation optimizes the availability and continuity of the SIS. DFMC SBAS MOPS requirement assures that the user has the CCE set currently being augmented still stored in the memory and does not undesirably replace it with a newer set. DFMC SARPs assure that the user receiver has sufficient time to decode the new CCE set before the system selects it for augmentation.

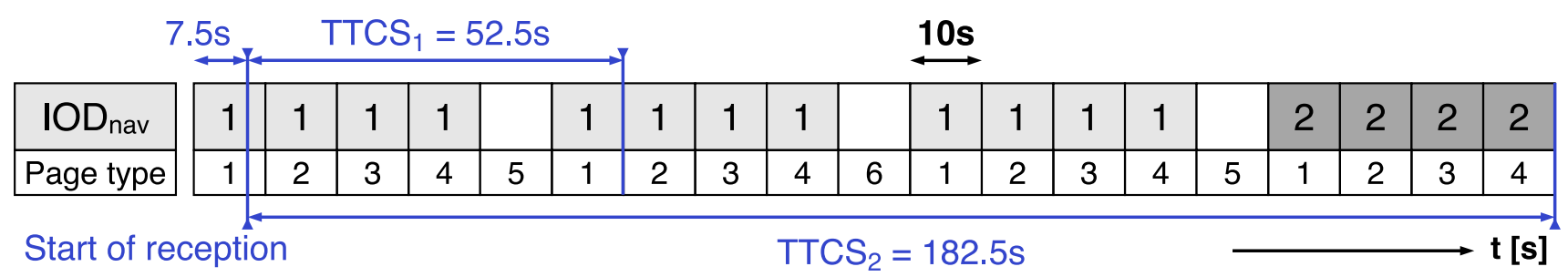

Fig. 4 Time needed to obtain $n$ unique and complete CCE sets $\left(\right.$ TTCS $\left._{n}\right)$ from a hypothetical F/NAV navigation data stream for $n=1$ and $n=2$ under the typical start of the reception beginning arbitrarily $7.5 \mathrm{~s}$ after the start of page type 1 
To statistically describe a given minimum TTCS $_{n}$, the probability of experiencing the minimum reception interval $P_{\mathrm{occ}}^{(n)}$ is proposed to be determined as:

$P_{\text {occ }}^{(n)}=P\left(\operatorname{TTCS}_{n}<\min \left(\operatorname{TTCS}_{n}\right)+\gamma\right)$

where $\gamma$ is the duration of the element of the given navigation message, which is $10 \mathrm{~s}$ for F/NAV and $6 \mathrm{~s}$ for LNAV.

\section{Maximum time to first complete clock correction and ephemeris sets}

The maximum time needed to obtain the first CCE set identifies the worst case that would delay the time needed to determine the clock and ephemeris of a given SV after the receiver is turned on. TTCS ${ }_{1}$ is a major contributor to the TTFF budget in cold and warm receiver starts (Paonni et al. 2010), making knowledge of its maximum relevant. The maximum TTCS $_{1}$ is usually assumed to depend only on the repetition interval of the CCE sets and the length of the decoding words as the same set and its IOD are considered to be continuously repeated in the navigation data stream (Paonni et al. 2010; Delépaut et al. 2020). An example of this assumption is shown in Fig. 5 (top) under the typical start of the F/NAV reception causing page type 1 to not be obtained. The assumption results in TTCS $_{1}$ being always less than the maximum TTCS $_{1}$ of $60 \mathrm{~s}$ for F/NAV and $36 \mathrm{~s}$ for LNAV. However, two additional contributors are of concern during operations that have not yet been considered.
The first contributor includes the IOD updates. If the user receiver starts receiving the navigation data during the transmission of the set and the IOD is updated instead of repeated, it has to collect a new set, introducing an additional delay. An example of the IOD $_{\text {nav }}$ update affecting the typical start of the F/NAV reception is shown in Fig. 5 (middle). After successfully receiving page types 2,3 , and 4 with $\operatorname{IOD}_{\text {nav }} 1$, an update of the $\mathrm{IOD}_{\text {nav }}$ parameter occurs. Since $\mathrm{IOD}_{\text {nav }}$ 1 is discontinued, the user receiver is required to wait until all four page types with the new $\mathrm{IOD}_{\text {nav }} 2$ are collected to obtain a complete set. During the IOD update, TTCS $_{1}$ is always less than the maximum TTCS of $_{1} 90 \mathrm{~s}$ for the F/NAV message.

The second contributor includes the atypical navigation message structures, as these may also introduce additional delays when obtaining a complete set. An example of hypothetical F/NAV subframes with mismatched IOD $_{\text {nav }}$ parameters affecting the typical start of the reception is shown in Fig. 5 (bottom). After successfully receiving page types 2,3 , and 4 with $\mathrm{IOD}_{\text {nav }} 1$, two subframes with mismatched $\mathrm{IOD}_{\text {nav }} 2$ and $\mathrm{IOD}_{\text {nav }} 3$ are transmitted. The mismatches make it impossible for the receiver to complete any CCE set until it manages to collect all necessary elements, which happens first for $\mathrm{IOD}_{\text {nav }} 3$. TTCS is always less than the maximum TTCS $_{1}$ of $170 \mathrm{~s}$ in the given example.

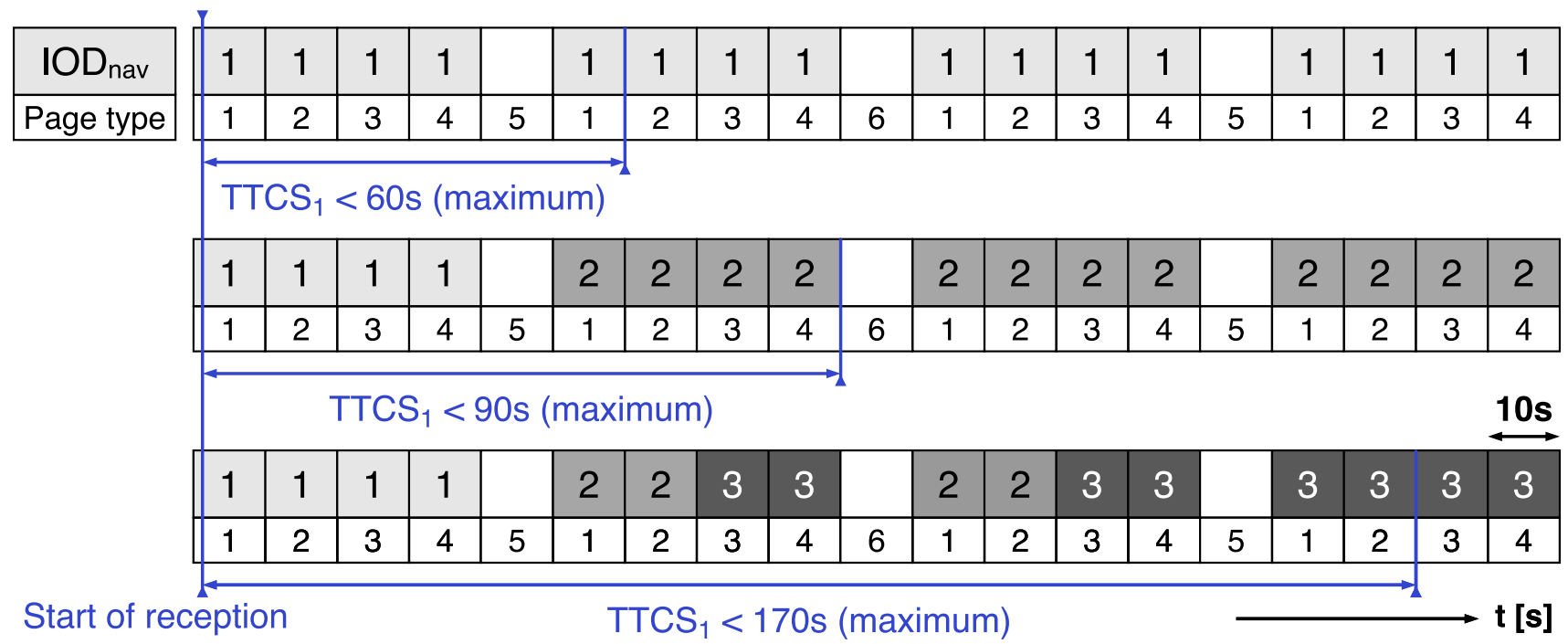

Fig. 5 Maximum time needed to obtain the first complete CCE set $\left(\mathrm{TTCS}_{1}\right)$ from a hypothetical F/NAV message stream under the typical start of the reception during the repetition of $\mathrm{IOD}_{\text {nav }} 1$ (top), update of $\mathrm{IOD}_{\text {nav }} 1$ to $\mathrm{IOD}_{\text {nav }} 2$ (middle), and subframes with arbitrar- ily mismatched $\mathrm{IOD}_{\text {nav }}$ parameters 2 and 3 (bottom). The maximum TTCS $_{1}$ is the upper bound of the worst possible TTCS 1 in a given stream 


\section{Sources of Broadcast Navigation Message Streams}

The study of the distribution of the CCE parameters using the proposed evaluation metrics requires detailed visibility of the binary content of the navigation message stream transmitted on the SIS. The GNSS community uses RINEX navigation files (RTCM-SC104 2018) to exchange the navigation messages broadcast by SVs. This format is also frequently used for accuracy and integrity assessments. However, the RINEX navigation file format lacks essential information needed for in-depth binary analyzes. To obtain the full binary information, proprietary formats of receiver manufacturers or alternative formats as the one proposed by Walter et al. (2016) that allow logging raw binary navigation data streams as outputted by the receiver tracking loops, have to be used.

\section{Limitations of RINEX navigation files}

Limitations of RINEX navigation files that constrain adequate visibility of the navigation data stream are identified as follows.

The first limitation includes the ambiguous time of transmission (ToT) and CCE parameters. The edge to which the ToT tags are rounded may vary between the different receivers or file converters. The rounding may depend on how the bit edges of the message structures are interpreted or when the receiver successfully decodes the CCE set for the first time. In addition, the CCE parameters might be reported with slightly different values, which may also vary among receivers due to the parameter conversion. Although these ambiguities may be partially mitigated by employing complex consolidation methods (Heng et al. 2012a; Rouch et al. 2015), these methods only operate on a certain level of confidence and may still fail to give the true transmitted CCE sets.

The second limitation is the ambiguous SIS health status. The SIS health status of the navigation message can change regardless of the IOD updates, which may not be properly reported in the RINEX navigation file after the conversion process. Further, the SIS health status may depend on immediate mechanisms not reported in the RINEX navigation file. For example, the health status of the LNAV message depends on the mismatched IODE parameters or failed parity checks on successive words (U.S. Department of Defense 2020).

The third limitation is the missing binary content. RINEX navigation files contain complete CCE sets once the receiver collects all necessary elements. However, there is no insight into the SIS content in-between the reported CCE sets and no insight into the detailed binary element structure used to obtain the reported CCE sets.

The third limitation is of concern when the atypical navigation message structures need to be assessed. An example of successive F/NAV subframes with mismatched IOD $_{\text {nav }}$ parameters occurring on the operational navigation data stream broadcast by the satellite with the SV identifier (SVID) 24 on May 6, 2017, is shown in Fig. 6 (left). A complete F/NAV CCE set could not have been obtained from the SIS for $1350 \mathrm{~s}$ after 15:34:10 due to the F/NAV subframes containing mismatched $\mathrm{IOD}_{\text {nav }} 59$ and 60 . To highlight the limitation, the content of the broadcast RINEX navigation file reporting the impacted period is shown in Fig. 6 (right). The broadcast RINEX navigation file was downloaded from the public server (CDDIS 2020). Indeed, the RINEX navigation file correctly reports the complete CCE sets tagged by $\mathrm{IOD}_{\text {nav }} 57$ and 62 . However, the file does not show the incomplete CCE sets tagged by $\mathrm{IOD}_{\text {nav }} 59$ and 60 . Furthermore, the RINEX navigation file does not indicate in any way that a complete CCE set was unobtainable from the SIS for over $20 \mathrm{~min}$, which could have rendered the SV unavailable for users requiring a new CCE set during that time.

Figure 6 also shows the limitation of the ambiguous ToT. The ticks on the time axis of the data stream are aligned to the start of the first bit of the first F/NAV page in the F/NAV subframe. However, the ToT reported in the RINEX file is
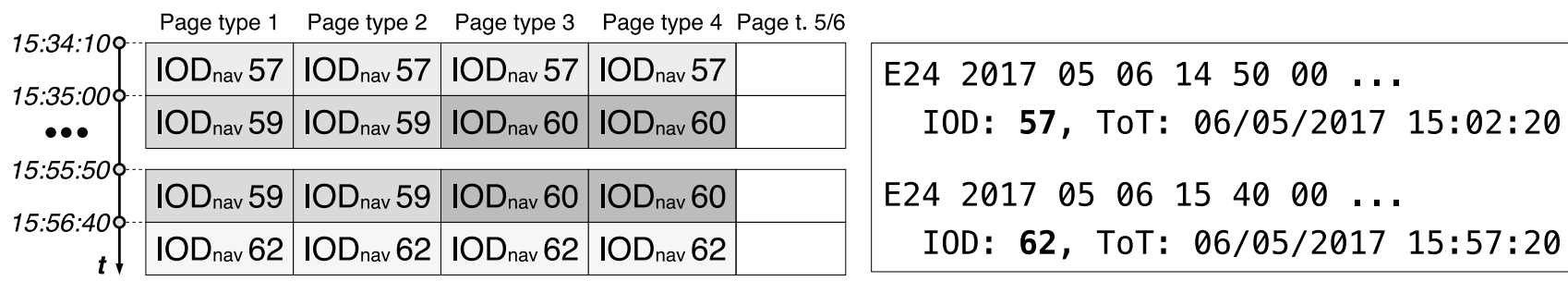

Fig. 6 (Left) Operational navigation stream broadcast by the Galileo SVID 24 on May 6, 2017. The rows of the vertical time axis represent the transmitted F/NAV subframes, showing the $\mathrm{IOD}_{\text {nav }}$ parameter of each page. The three dots indicate that all subframes transmitted between 15:35:00 and 15:56:40 contain the same pattern of mis- matched $\mathrm{IOD}_{\text {nav }}$ parameters 59 and 60. (Right) CCE sets reported in the broadcast RINEX navigation file for the period of the navigation stream. The RINEX navigation file does not reveal the incomplete CCE sets tagged by $\mathrm{IOD}_{\text {nav }} 59$ and 60 , and does not indicate that a complete set is unobtainable from the SIS for over $20 \mathrm{~min}$ 


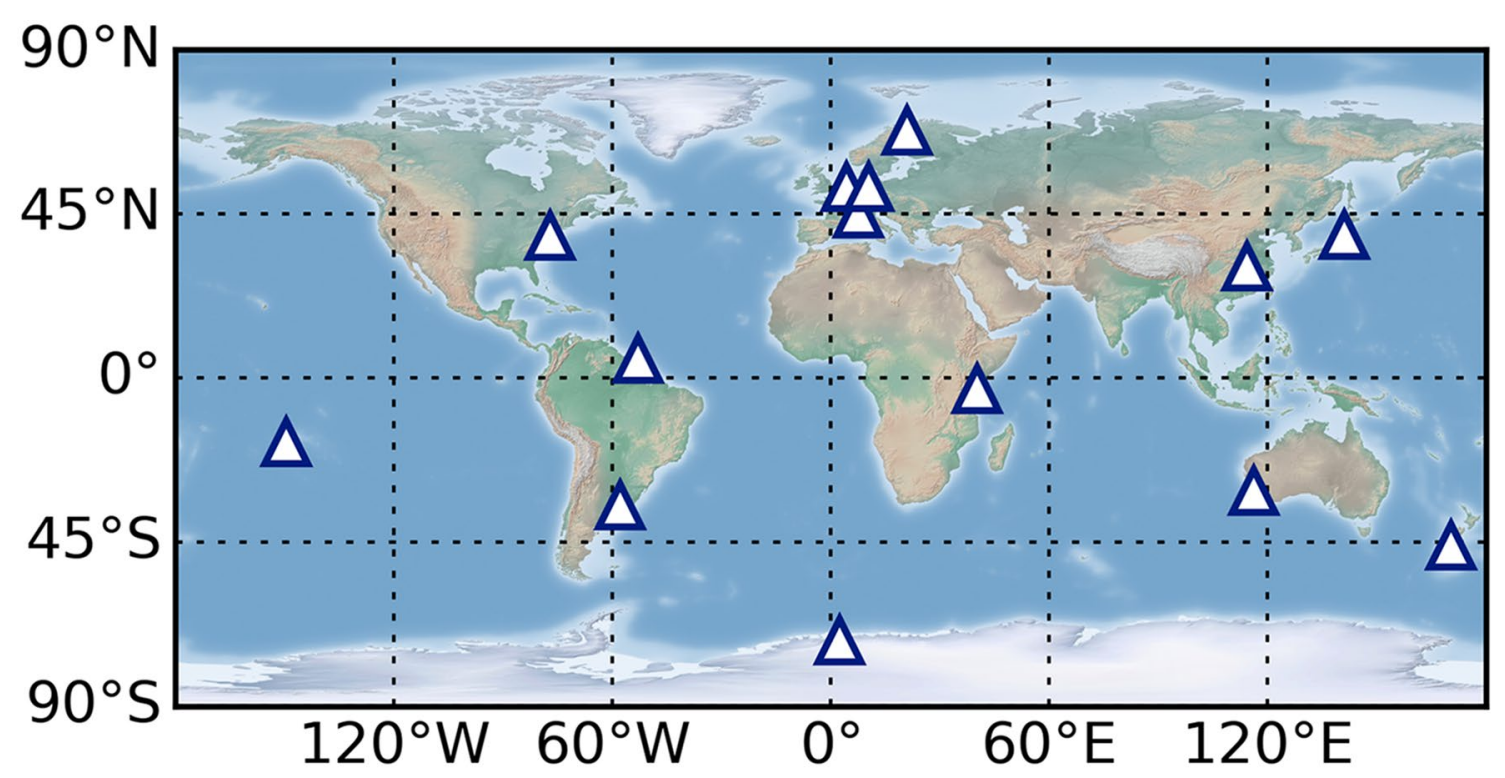

Fig. 7 Worldwide deployment of the stations of the GESS network as of January 2018

in this case aligned to the end of the last bit of the CCE set, resulting in the timestamp 15:57:20 for $\mathrm{IOD}_{\text {nav }} 62$ that differs from the timestamp 15:56:40 on the time axis.

\section{Binary navigation data}

Most GNSS receiver manufacturers allow configuring their receivers to $\log$ and store the collected binary navigation data streams in proprietary formats. The Galileo Performance Center, located at the premises of the European Space Agency (ESA), hosts a so-called Time and Geodetic Validation Facility (TGVF), which comprises a worldwide network of Galileo experimental sensor stations (GESS) that are capable of receiving Galileo E1/E5a and GPS L1/ L5 signals. The network employs, among others, Septentrio receivers that collect the bits of tracked GPS F/NAV and GPS LNAV data streams in a proprietary binary file structure called Septentrio binary format (SBF) (Septentrio 2017). The worldwide deployment of the GESS network is as of January 2018, shown in Fig. 7.

\section{Analysis of broadcast navigation messages using evaluation metrics}

To study the distribution of the CCE parameters, the operational broadcast Galileo F/NAV and GPS LNAV messages are analyzed using the proposed evaluation metrics. Three and a half years of binary data, from January 1, 2017, until June 30, 2020, collected by the Septentrio receivers of the GESS network, are processed. The data start date is chosen to be after the declaration of Galileo's initial services on December 15, 2016, to have representative results for both navigation messages. The service incident period of Galileo from July 10, 2019, to July 17, 2019, is excluded from the processing to avoid the CCE sets that were not updated for long periods. The processing steps are as follows: (i) error correction and extraction of navigation message elements, (ii) determination of the SIS health status of each element, and (iii) consolidation of the binary navigation data streams from all stations into a single broadcast navigation stream. The study considers arbitrary and healthy-only SIS health statuses. The arbitrary health status includes healthy, unhealthy, and marginal statuses.

In total, over 218 million F/NAV pages and over 590 million LNAV subframes are identified in the data set. The number of missing and discarded elements is summarized in Table 2. The data gap is determined when an element

Table 2 Number of navigation message elements of Galileo F/NAV and GPS LNAV messages identified in the evaluated data set along with the number of data gaps and the number of discarded, consolidated, and healthy-only elements

\begin{tabular}{lll}
\hline Navigation message element & F/NAV page & LNAV subframe \\
\hline Identified elements & $218,879,113$ & $590,687,626$ \\
$\begin{array}{l}\text { Data gaps } \\
\text { Discarded elements (ambiguous }\end{array}$ & 552,503 & $4,399,584$ \\
$\quad$ content) & & 694 \\
$\begin{array}{l}\text { Discarded elements (single station) } \\
\text { Consolidated elements }\end{array}$ & Not applied & $4,026,228$ \\
Healthy-only elements & $218,326,608$ & $582,261,120$ \\
& $187,986,424$ & $562,794,974$
\end{tabular}


Table 3 Probability of occurrence of structures with mismatched IOD parameters of Galileo F/NAV and GPS LNAV messages for the arbitrary SIS health status

\begin{tabular}{lllrr}
\hline Structure & $\begin{array}{l}\text { Probability of occur- } \\
\text { rence }\end{array}$ & $\begin{array}{l}\text { Number of structures with } \\
\text { mismatches }\end{array}$ & $\begin{array}{l}\text { Total number of } \\
\text { structures }\end{array}$ & Last observed mismatch \\
\hline Galileo F/NAV subframe & $3.09 \times 10^{-6}$ & 135 & $43,665,191$ & SVID 25, May 15, 2020 09:34:10 \\
GPS LNAV frame & $2.11 \times 10^{-4}$ & 24,558 & $116,404,495$ & PRN 11, June 30, 2020 22:57:00 \\
\hline
\end{tabular}

\begin{tabular}{|c|c|c|c|c|}
\hline \multirow{2}{*}{ 14:53:000 } & Subframe 1 & Subframe 2 & Subframe 3 & Subframes 4 and 5 \\
\hline & IODE 42 & IODE 42 & IODE 42 & \\
\hline & IODE 42 & IODE 7 & IODE 7 & \\
\hline & IODE 7 & IODE 7 & IODE 7 & \\
\hline
\end{tabular}

Fig. 8 Operational navigation stream broadcast by the GPS SV PRN 12 on June 30, 2020. The rows of the vertical time axis represent the transmitted LNAV frames, showing the IODE parameter of each subframe. At 14:53:30, a frame whose subframes 2 and 3 contain mismatched IODE 7 instead of IODE 42 is transmitted

should have been received but is not recorded by any station or when the error checking fails. The ambiguous content filter is triggered when the binary content is not unanimously confirmed by all stations monitoring the SIS. The single station filter is triggered when the SIS is monitored by only one station. The single station filter is only applied to the LNAV message due to its lower robustness to the burst bit errors. After the consolidation step, over 218 million F/NAV pages and 582 million LNAV subframes with arbitrary SIS

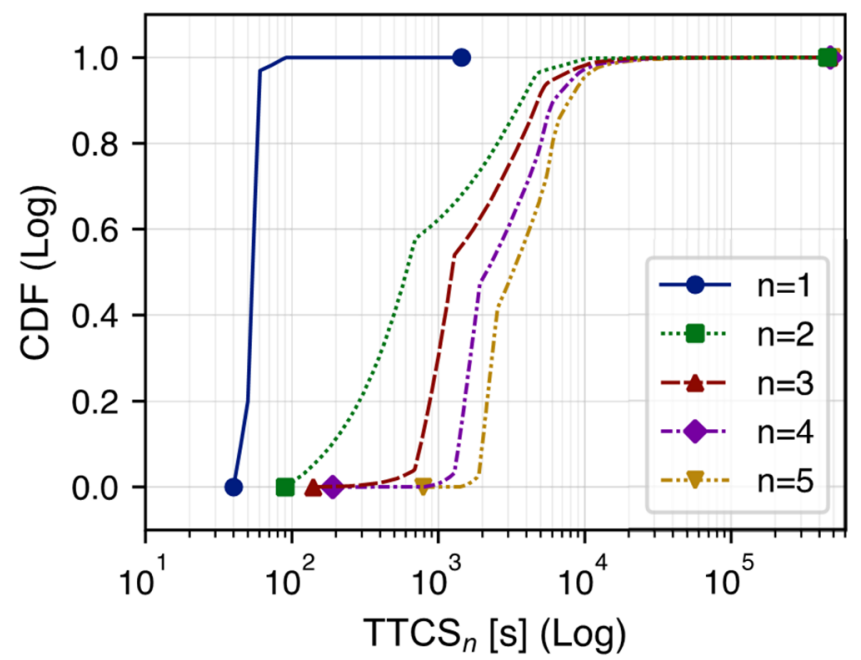

Fig. 9 Cumulative density functions of the times needed to obtain $n$ unique and complete CCE sets (TTCS ${ }_{n}$ ) of the F/NAV message (left) and LNAV message (right) for the arbitrary SIS health status under the uniformly distributed start of the reception. $\operatorname{TTCS}_{n}$ is included health statuses remain. The extracted data are equivalent to over 69 years of operational Galileo E5a SIS and 110 years of operational GPS L1C/A SIS, which is a sufficient period for the study.

\section{Atypical navigation message structures}

The observed structures with mismatched IOD parameters of both evaluated navigation messages are summarized in Table 3 for the arbitrary SIS health status. As can be seen, the mismatched IODE parameters are quite common for the LNAV message with the probability of occurrence $P_{\text {occ }}=2.11 \times 10^{-4}$. This is attributed to the data set cutovers as the IODE mismatches occur in the LNAV subframes 2 and 3 belonging to the new CCE set, while the LNAV subframe 1 still belongs to the old set. An example of such a frame transmitted by the SV with the pseudo-random noise (PRN) number 12 on June 30, 2020, is shown in Fig. 8, where subframe 1 contains IODE 42 and subframes 2 and 3 contain a mismatched IODE 7 . Considering the interface specification is not explicit in which cutovers are to be considered normal and which not (U.S. Department of Defense

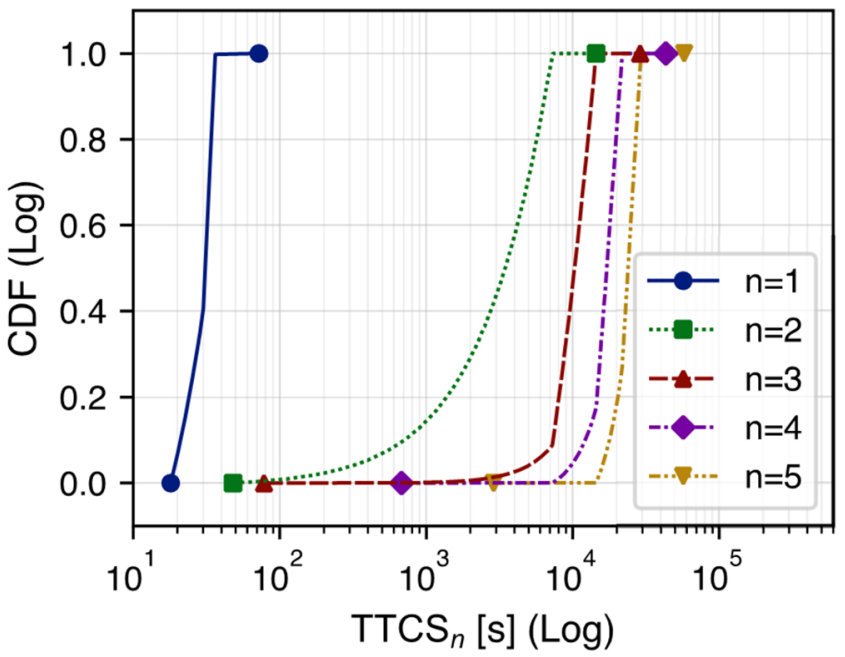

regardless of the SIS health status (healthy, unhealthy, marginal) during the period needed to obtain $n$ sets. The maxima and minima of $\mathrm{TTCS}_{n}$ are indicated with markers as these are relevant to the receiver. Other values of CDFs equal to 0 and 1 are not shown 


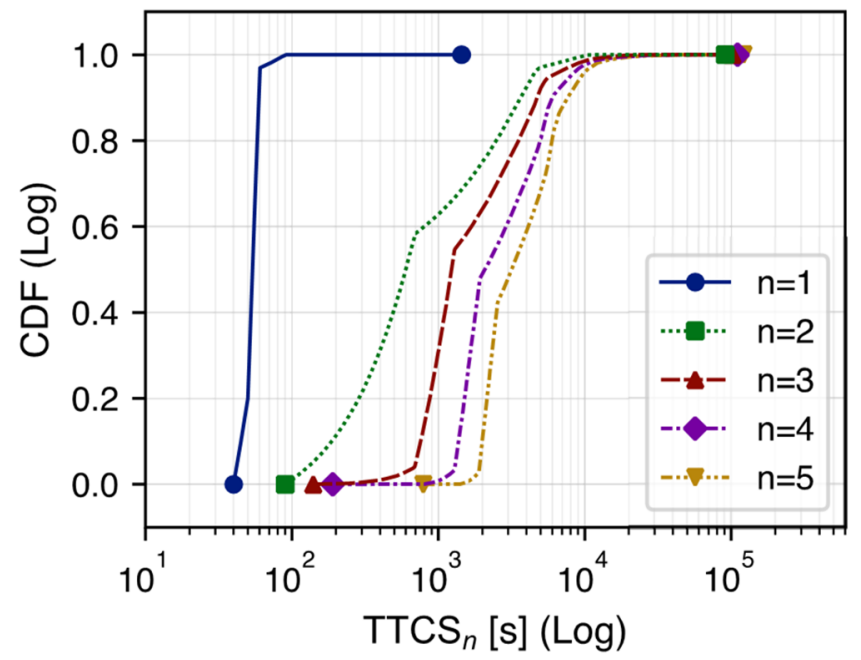

Fig. 10 Cumulative density functions of the times needed to obtain $n$ unique and complete CCE sets $\left(\right.$ TTCS $_{n}$ ) of the F/NAV message (left) and LNAV message (right) for the healthy-only SIS under the uniformly distributed start of the reception. TTCS $_{n}$ is included only

Table 4 Minima of TTCS $_{n}$ and related probabilities of experiencing minimum reception intervals for the arbitrary SIS health status

\begin{tabular}{llllll}
\hline$n$ & \multicolumn{2}{l}{$\mathrm{TTCS}_{n}$ of F/NAV } & & \multicolumn{2}{l}{ TTCS $_{n}$ of LNAV } \\
\cline { 2 - 3 } \cline { 5 - 6 } & Min $[\mathrm{s}]$ & $P_{\text {occ }}^{(n)}$ & & Min $[\mathrm{s}]$ & $P_{\text {occ }}^{(n)}$ \\
\hline 1 & 40 & $2.00 \times 10^{-1}$ & & 18 & $2.00 \times 10^{-1}$ \\
2 & 90 & $1.03 \times 10^{-2}$ & & 48 & $8.75 \times 10^{-4}$ \\
3 & 140 & $1.38 \times 10^{-4}$ & & 78 & $1.98 \times 10^{-7}$ \\
4 & 190 & $2.93 \times 10^{-7}$ & & 678 & $2.00 \times 10^{-9}$ \\
5 & 790 & $2.29 \times 10^{-7}$ & & 2886 & $2.07 \times 10^{-9}$ \\
\hline
\end{tabular}

2013), the LNAV frames impacted by the mismatches remain also included in the healthy-only SIS analysis, resulting in the probability of occurrence $P_{\text {occ }}=2.13 \times 10^{-4}$. The probability of occurrence of the F/NAV subframes with mismatched $\mathrm{IOD}_{\text {nav }}$ parameters is $P_{\text {occ }}=3.09 \times 10^{-6}$ for the arbitrary health status and $P_{\text {occ }}=3.48 \times 10^{-6}$ for the healthy-only SIS. The $\mathrm{IOD}_{\text {nav }}$ mismatches are rare and uncorrelated, occurring on a single $\mathrm{SV}$ at a given time. One such transmission, lasting $1350 \mathrm{~s}$ on May 6, 2017, is shown in Fig. 6.

Neither structures with missing element numbers nor structures with wrongly ordered elements are observed for either evaluated message. However, this might change for the Galileo F/NAV message along with the evolution of the system without violating the current interface commitments. The user is requested always to decode the page type identifier.

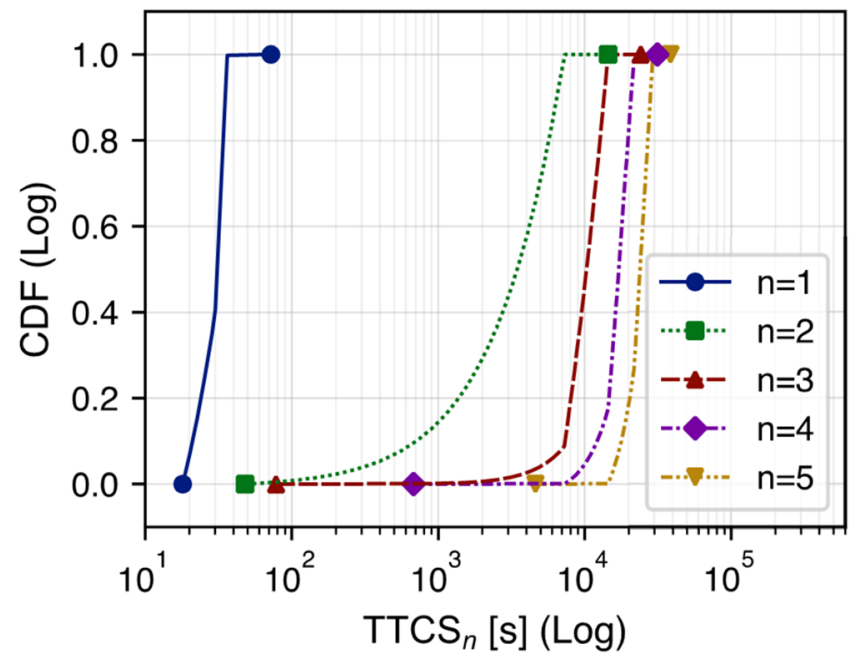

when the given SIS is healthy for the whole period needed to obtain $n$ sets. The maxima and minima of TTCS $_{n}$ are indicated with markers as these are relevant to the receiver. Other values of CDFs equal to 0 and 1 are not shown

\section{Mitigation actions for mismatched F/NAV subframes}

Possible root causes leading to the F/NAV subframes with mismatched $\mathrm{IOD}_{\text {nav }}$ parameters, such as the event on the SVID 24 shown in Fig. 6, are identified at the Galileo system level. Appropriate mitigation actions have been established to prevent the reoccurrence of such events in Galileo after the full operational capability (FOC) declaration.

\section{Time to complete clock correction and ephemeris sets}

The $\operatorname{TTCS}_{n}$ metric is evaluated for $n \in\{1,2,3,4,5\}$ complete CCE sets. The starting point of the reception is assumed to be random and uniformly distributed along the navigation data stream. The cumulative density functions (CDFs) of TTCS of both evaluated messages are shown for the arbitrary SIS health status in Fig. 9, and the healthy-only SIS in Fig. 10.

Figures 9 and 10 show that the statistical distributions of TTCS $_{n}$ are almost identical for the arbitrary and healthy-only SIS health statuses. The only notable difference is between the maxima of TTCS $_{n}$ for $n>1$. Unhealthy and marginal SIS health statuses are characterized by lengthier periods during which IOD parameters are not updated, resulting in larger maxima of TTCS $_{n}$ in Fig. 9. The minimum time to multiple CCE sets and the maximum time to the first CCE set are discussed in separate sections.

\section{Minimum time to multiple complete clock correction and ephemeris sets}

The minima of $\mathrm{TTCS}_{n}$ from Fig. 9 are summarized for F/NAV and LNAV messages in Table 4 . Table 4 also 
Table 5 Yearly and overall medians, 99th percentiles, and maxima of the statistical distributions of the times needed to obtain the first complete CCE set $\left(\right.$ TTCS $\left._{1}\right)$ of the Galileo F/NAV and GPS LNAV messages for the arbitrary SIS health status

\begin{tabular}{|c|c|c|c|c|c|c|}
\hline \multirow[t]{2}{*}{ Year } & \multicolumn{3}{|c|}{$\mathrm{TTCS}_{1}$ of F/NAV } & \multicolumn{3}{|c|}{ TTCS $_{1}$ of LNAV } \\
\hline & Median [s] & $99 \%[s]$ & $\operatorname{Max}[\mathrm{s}]$ & Median [s] & $99 \%[\mathrm{~s}]$ & $\operatorname{Max}[\mathrm{s}]$ \\
\hline 2020 & 53.90 & 80.23 & 150.00 & 31.00 & 35.92 & 72.00 \\
\hline 2019 & 53.90 & 80.21 & 980.00 & 31.00 & 35.92 & 72.00 \\
\hline 2018 & 53.90 & 80.51 & 340.00 & 31.00 & 35.92 & 72.00 \\
\hline 2017 & 53.90 & 80.11 & 1440.00 & 31.00 & 35.92 & 72.00 \\
\hline All & 53.90 & 80.27 & 1440.00 & 31.00 & 35.92 & 72.00 \\
\hline $\begin{array}{l}\text { All (healthy- } \\
\text { only SIS) }\end{array}$ & 53.90 & 80.36 & 1440.00 & 31.00 & 35.92 & 72.00 \\
\hline
\end{tabular}

summarizes the probabilities of experiencing the minimum reception intervals $P_{\text {occ }}^{(n)}$ determined using Eq. (1). The results show that the F/NAV message can transmit four different CCE sets in four successive subframes as the minimum TTCS $_{4}$ is $190 \mathrm{~s}$, confirming the existence of the fast double updates of the $\mathrm{IOD}_{\text {nav }}$ parameter (Lapin et al. 2018). The results of Table 4 are particularly relevant to SBAS. DFMC SBAS MOPS assumes that there can be at most four F/NAV and at most three LNAV CCE sets for a single SV in any given interval of five minutes (Eurocae 2021), implying a minimum capacity of the receiver memory. Considering that five F/NAV CCE sets occur earliest in $790 \mathrm{~s}$ and four LNAV CCE sets in $678 \mathrm{~s}$ for the arbitrary SIS health status, the requirements in DFMC SBAS MOPS are in line with both evaluated messages.

\section{Maximum time to first complete clock correction and ephemeris set}

Figure 9 shows that the maximum TTCS $_{1}$ during the arbitrary SIS health status is $1440 \mathrm{~s}$ for the F/NAV message and $72 \mathrm{~s}$ for the LNAV message. Figure 10 shows that the respective maxima remain the same for the healthy-only SIS. The maxima of both messages are higher than the usual assumptions of $60 \mathrm{~s}$ for F/NAV and $36 \mathrm{~s}$ for LNAV. The higher values are caused by the IOD updates and atypical navigation message structures whose impacts on maximum TTCS $_{1}$ are shown in Fig. 5.

The impact of the IOD updates on $\mathrm{TTCS}_{1}$ depends on the update periods. Short IOD update periods translate into a higher probability of experiencing a delay when obtaining the first complete CCE set as the unique sets are updated more frequently. The F/NAV message has shorter IOD update periods with a median value of $600 \mathrm{~s}$ than the LNAV message with a median value of $7200 \mathrm{~s}$ (Lapin et al. 2018). Shorter IOD update periods shift the body of the statistical distribution of TTCS ${ }_{1}$ to higher values, as is shown in Figs. 9 and 10 .

The impact of the atypical message structures on TTCS $_{1}$ is relevant mostly for the Galileo F/NAV message as it may experience rarely successive subframes with mismatched
$\mathrm{IOD}_{\text {nav }}$ parameters that do not allow completing any CCE set for long periods. Consequently, the statistical distributions of TTCS ${ }_{1}$ of F/NAV have noticeable tails, as is shown in Figs. 9 (left) and 10 (left). In the case of the LNAV message, no lengthy periods of frames with mismatched IODE parameters are observed and the related statistical distributions of TTCS $_{1}$ show no significant tails.

The results indicate that it is better to describe TTCS $_{1}$ using percentiles instead of maxima. The medians, 99th percentiles, and maxima of TTCS $_{1}$ of both navigation messages are for each evaluated year summarized in Table 5 for the arbitrary SIS health status. Table 5 also shows the overall percentiles for the arbitrary and healthy-only SIS health statuses. As can be seen, the maxima of the F/NAV message differ each year and do not follow any stable pattern. However, the medians and 99th percentiles remain stable along with the evaluated years and can be used to describe $\mathrm{TTCS}_{1}$. In the case of the LNAV message, the medians and 99th percentiles also remain stable along with the years. The values do not significantly differ between the evaluated health statuses in either message. For simplicity, the values of 80 and $36 \mathrm{~s}$ are proposed to be taken as the conservative times needed to obtain the first F/NAV and LNAV set during operations, respectively. These values correspond to the duration of $8 \mathrm{~F} / \mathrm{NAV}$ pages and $6 \mathrm{LNAV}$ subframes. The full impact of TTCS $_{1}$ on the TTFF depends on whether the identified contributors occur on multiple SVs simultaneously as complete sets of several SVs are needed for a position fix. The correlation of the CCE set updates between multiple SVs was not investigated.

\section{Conclusions}

The distribution of the CCE parameters in broadcast Galileo F/NAV and GPS LNAV messages was studied. The evaluation metrics were proposed and used to analyze three and a half years of binary navigation data. The IOD update periods and structures with mismatched IOD parameters are shown to be important contributors to the time needed to obtain the first CCE set during operations. The message timing 
requirements of DFMC SBAS MOPS are in line with both evaluated navigation messages as there can be indeed at most four F/NAV and at most three LNAV complete CCE sets in any given interval of five minutes. The achieved results are relevant to a wide range of GNSS applications that depend on the CCE sets broadcast in navigation messages, especially when optimizing the SIS availability and continuity.

Acknowledgements The authors would like to thank the Galileo Performance Center for providing the binary data.

Availability data and materials The binary data of the Time and Geodetic Validation Facility (TGVF) used in this study belong to the Galileo Performance Center and can be requested at https://www.tgvf.esa. int/data-request-form/.

Open Access This article is licensed under a Creative Commons Attribution 4.0 International License, which permits use, sharing, adaptation, distribution and reproduction in any medium or format, as long as you give appropriate credit to the original author(s) and the source, provide a link to the Creative Commons licence, and indicate if changes were made. The images or other third party material in this article are included in the article's Creative Commons licence, unless indicated otherwise in a credit line to the material. If material is not included in the article's Creative Commons licence and your intended use is not permitted by statutory regulation or exceeds the permitted use, you will need to obtain permission directly from the copyright holder. To view a copy of this licence, visit http://creativecommons.org/licenses/by/4.0/.

\section{References}

CDDIS (2020) Global data archive of IGS: Crustal Dynamics Data Information System (CDDIS). ftp://cddis.gsfc.nasa.gov/pub/gps/data/daily/

Cohenour C, van Graas F (2011) GPS orbit and clock error distributions. Navigation 58(1):17-28. https://doi.org/10.1002/j.21614296.2011.tb01789.x

Delépaut A, Giordano P, Ventura-Traveset J, Blonski D, Schönfeldt M, Schoonejans P, Aziz S, Walker R (2020) Use of GNSS for lunar missions and plans for lunar in-orbit development. Adv Space Res 66(12):2739-2756. https://doi.org/10.1016/j.asr.2020.05.018

Eurocae (2021) ED-259A: minimum operational performance specification for Galileo/global positioning system / satellite-based augmentation system airborne equipment, issue 0.8 (draft)

European Union (2020) European GNSS (GALILEO) Open servicesignal-in-space interface control document, issue 2.0, December. https://www.gsc-europa.eu/sites/default/files/sites/all/files/Galil eo_OS_SIS_ICD_v2.0.pdf

European Union (2019) European GNSS (GALILEO) open service-service definition document, issue 1.1, May. https://www.gsc-europa. $\mathrm{eu} /$ sites/default/files/sites/all/files/Galileo-OS-SDD_v1.1.pdf

Gunning K, Walter T, Enge P (2015) Characterization of GLONASS broadcast clock and ephemeris: nominal performance and fault trends for ARAIM. In: Proc. ION ITM 2017, Monterey, CA, USA, January 30-2, pp 170-183. https://doi.org/10.33012/2017.14926

Heng L, Gao GX, Walter T, Enge P (2012a) GPS signal-in-space integrity performance evolution in the last decade. IEEE Trans Aerosp Electron Syst 48(4):2932-2946. https://doi.org/10.1109/TAES.2012.6324670

Heng L, Gao GX, Walter T, Enge P (2012b) Statistical characterization of GLONASS broadcast clock errors and signal-in-space errors. In: Proc. ION ITM 2012, Newport Beach, CA, USA, January $30-1$, pp 1697-1707

ICAO (2018) DFMC SARPs, Part B, JWGs6 Flimsy 33, DS2/WP/3 v2.2 (draft)

Kaplan E, Hegarty C (2017) Understanding GPS/GNSS: principles and applications, 3rd edn. Artech House

Lapin I, Samson J, Wallner S, Mabilleau M, Durel P (2018) Impact of issue of data update periods on satellite-based augmentation systems. In: Proceedings of the 2018 9th ESA workshop on satellite navigation technologies and european workshop on GNSS signals and signal processing (NAVITEC), pp 1-10. https://doi. org/10.1109/NAVITEC.2018.8642649

Lv Y, Geng T, Zhao Q, Xie X, Zhou R (2019) Initial assessment of BDS-3 preliminary system signal-in-space range error. GPS Solut 24(16):1-13. https://doi.org/10.1007/s10291-019-0928-x

Montenbruck O, Steigenberger P, Hauschild A (2018) Multi-GNSS signal-in-space range error assessment-methodology and results. Adv Space Res 61(12):3020-3038. https://doi.org/10. 1016/j.asr.2018.03.041

Paonni M, Anghileri M, Wallner S, Avila-Rodriguez JA, Eissfeller B (2010) Performance assessment of gnss signals in terms of time to first fix for cold, warm and hot start. In: Proc. ION ITM 2010, San Diego, CA, USA, January 25-27, pp 1051-1066

Perea S, Meurer M, Rippl M, Belabbas B, Joerger M (2017) URA/ SISA analysis for GPS and Galileo to support ARAIM. Navigation 64(2):237-254. https://doi.org/10.1002/navi.199

Rouch C, Bonhoure B, Suard N, Mabilleau M, Vuillaume J, Dulery C, Lapeyre D, Sauce C (2015) Galileo ephemeris consolidation and control analysis-GECCO. In: Proc. ION GNSS 2015, Tampa, FL, USA, September 14-18, pp 1947-1957

RTCM-SC104 (2018) RINEX the receiver independent exchange format, version 3.04, International GNSS Service (IGS), RINEX Working Group and Radio Technical Commission for Maritime Services Special Committee 104 (RTCM-SC104), November 23. http://acc.igs.org/misc/rinex304.pdf

Septentrio (2017) PPSDK SBF reference guide: applicable to PPSDK version 4.1.8, February 20

Steigenberger P, Montenbruck O, Hessels U (2015) Performance evaluation of the early CNAV navigation message. Navigation 62(3):219-228. https://doi.org/10.1002/navi.111

U.S. Department of Defense (2013) Interface specification IS-GPS200H, September. https://www.gps.gov/technical/icwg/IS-GPS200H.pdf

U.S. Department of Defense (2020) Global positioning system standard positioning service (SPS) - performance standard, 5th edn, April. https://www.gps.gov/technical/ps/2020-SPS-perfo rmance-standard.pdf

Walter T, Gunning K, Blanch J (2016) Improved ephemeris monitoring for GNSS. In: Proc. ION ITM 2016, Monterey, CA, USA, January 25-28, pp 600-608. https://doi.org/10.33012/2016.13414

Wang A, Chen J, Zhang Y, Wang J, Wang B (2019) Performance evaluation of the CNAV broadcast ephemeris. J Navig 72(5):13311344. https://doi.org/10.1017/S037346331900016X

Warren DL, Raquet JF (2003) Broadcast vs. precise GPS ephemerides: a historical perspective. GPS Solut 7:151-156. https://doi.org/10. 1007/s10291-003-0065-3

Wu W, Guo F, Zheng J (2020) Analysis of Galileo signal-in-space range error and positioning performance during 2015-2018. Satell Navig. https://doi.org/10.1186/s43020-019-0005-1

Wu Y, Liu X, Liu W, Ren J, Lou Y, Dai X, Fang X (2017) Long-term behavior and statistical characterization of BeiDou signal-in-space 
errors. GPS Solut 21(4):1907-1922. https://doi.org/10.1007/ s10291-017-0663-0

Publisher's Note Springer Nature remains neutral with regard to jurisdictional claims in published maps and institutional affiliations.

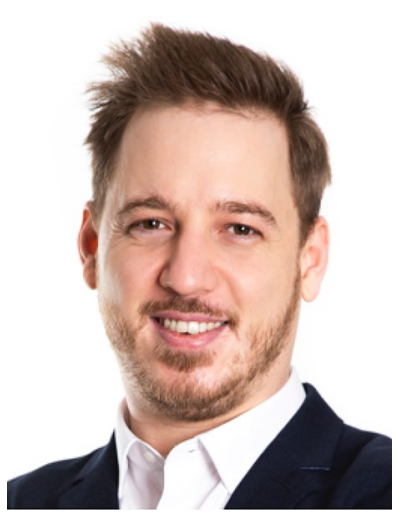

Ivan Lapin received his Ing. and $\mathrm{Ph}$.D. degrees in telecommunications from the Slovak University of Technology in Bratislava (Slovakia). He joined the European Space Agency (ESA) as an EGNOS System Performance Engineer in 2016. He is currently a research fellow at ESA working on hybrid GNSS/5G positioning, integrity, and performances of navigation systems (e-mail: ivan. lapin@esa.int, ORCID: 0000-0002-1847-5499).

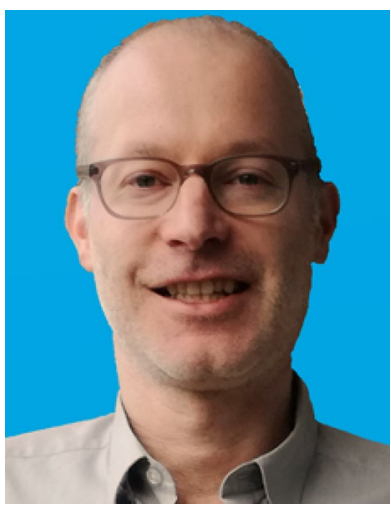

Jaron Samson holds an M.Scdegree in Physical and Mathematical Geodesy from Delft University of Technology (the Netherlands). He joined the European Space Agency (ESA) in 2003. Since 2012, he works as an EGNOS System Engineer at ESAГÇÖs EGNOS Project Office in Toulouse (France) (e-mail: jaron.samson@esa.int).

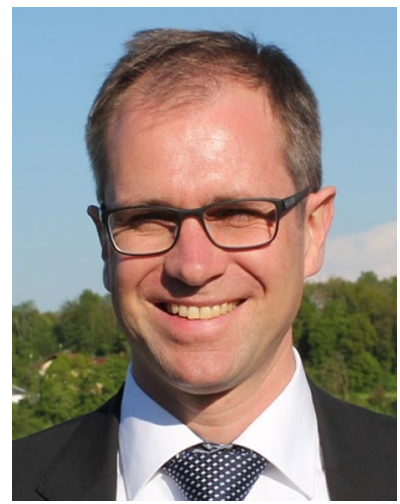

Stefan Wallner is the Head of the Galileo 1st Generation (G1) Signal in Space Engineering Unit within the Navigation Directorate at ESA/ESTEC. He is leading the Safety-of-Life Workplan activities at ESA level within the Galileo System Office with the objective to enable Galileo for augmentation by SBAS and for Galileo to contribute to H-ARAIM solutions (e-mail: stefan.wallner@esa.int).

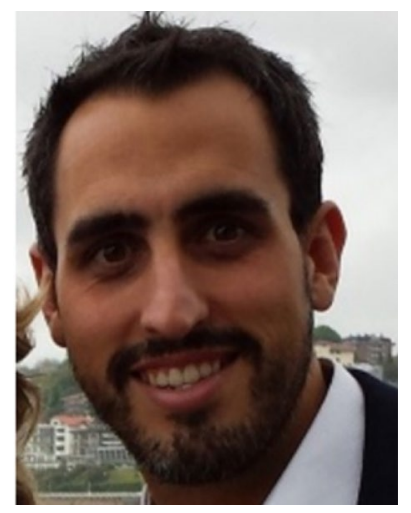

Carlos Lopez holds an M.Sc in Telecommunication Engineering and a Master in Space Technology both from the Polytechnic University of Madrid (Spain). Since 2005 he has been working in EGNOS, first at GMV (Spain) and since 2006 at ESA. He is currently the EGNOS System Performance Principal Engineer at ESAГÇÖs EGNOS Project Office in Toulouse (France) (e-mail: carlos.lopez@esa.int).

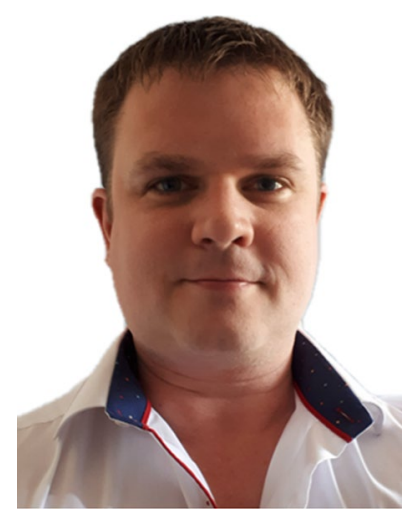

Mikael Mabilleau is a standardization engineer on SBAS working in the EGNOS exploitation team of the European GNSS Agency (GSA). Since his graduation as an engineer from the French civil aviation school (ENAC) in 2006, he has been involved in GNSS standardization activities carried by the main civil aviation standardization bodies: EUROCAE WG 62, RTCA SC 159 or ICAO NSP (e-mail: mikael.mabilleau@gsa. europa.eu). 